\title{
Charge and spin ordering in $\mathrm{Nd}_{1 / 3} \mathrm{Sr}_{2 / 3} \mathrm{FeO}_{3}$
}

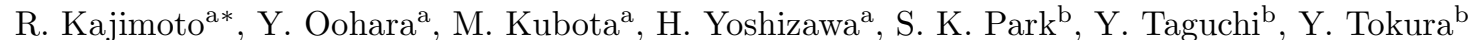 \\ ${ }^{a}$ Neutron Scattering Laboratory, I. S. S. P., University of Tokyo, Tokai, Ibaraki, 319-1106, Japan \\ ${ }^{\mathrm{b}}$ Department of Applied Physics, University of Tokyo, Bunkyo-ku, Tokyo 113-8656, Japan
}

(November 21, 2018)

\begin{abstract}
We have investigated the charge and spin ordering in $\mathrm{Nd}_{1 / 3} \mathrm{Sr}_{2 / 3} \mathrm{FeO}_{3}$ with neutron diffraction technique. This sample undergoes a charge ordering transition accompanying charge disproportionation of $2 \mathrm{Fe}^{4+} \rightarrow \mathrm{Fe}^{3+}+\mathrm{Fe}^{5+}$. We measured the superlattice reflections due to the charge and spin ordering, and confirmed that charges and spins order simultaneously at $T_{\mathrm{CO}}=185 \mathrm{~K}$. The ordering pattern of charges and spins in this sample can be viewed as three dimensional stripe order, and is compared with two dimensional stripe order observed in other transition metal oxides.
\end{abstract}

keywords: A. oxides, B. crystal growth, C. neutron scattering, D. charge-density wave, magnetic structure

Charge ordering is widely seen in hole doped transition metal oxides, such as cuprates (e.g. $\mathrm{La}_{2-x} \mathrm{Sr}_{x} \mathrm{CuO}_{4}[\mathrm{i} \mid$ ), nickelates (e.g. $\mathrm{La}_{2-x} \mathrm{Sr}_{x} \mathrm{NiO}_{4+\delta}$ [2]) and manganites (e.g. $\mathrm{Pr}_{1 / 2} \mathrm{Ca}_{1 / 2} \mathrm{MnO}_{3}$ 晒 and $\mathrm{Nd}_{1 / 2} \mathrm{Sr}_{1 / 2} \mathrm{MnO}_{3}$ 泃). Because the charge ordering transition occurs at the region near the insulator-metal transition or superconducting transition, a number of works concentrated on the study of this phenomenon in order to clarify a key to understand the origin of the insulator-metal transition or superconducting transition. From these studies, it is widely recognized that the charge ordering phenomenon is a consequence of the coupling or the competition among the degrees of freedom of charge, spin, lattice, or orbitals

Hole doped perovskite-type $R_{1 / 3} \mathrm{Sr}_{2 / 3} \mathrm{FeO}_{3}$ is one of the system which undergoes a charge ordering transition. In this system, the charge ordering accompanies charge disproportionation of $2 \mathrm{Fe}^{4+} \rightarrow \mathrm{Fe}^{3+}+\mathrm{Fe}^{5+}$ and simultaneous antiferromagnetic spin ordering. A pioneering work with Mössbauer spectroscopy on $\mathrm{La}_{1 / 3} \mathrm{Sr}_{2 / 3} \mathrm{Fe}_{3}$ by Takano et al. revealed that there are two kinds of Fe ions with the ratio of $2: 1$, and they were attributed to $\mathrm{Fe}^{3+}$ and $\mathrm{Fe}^{5+}[6]$. This charge disproportionation state was confirmed by Battle et al. with the neutron powder diffraction measurements on the same compound. They observed the antiferromagnetic spin ordering with sixfold periodicity along the cubic [111] direction and showed that this magnetic structure was generated from the ordering of the layers of the $\mathrm{Fe}^{3+}$ ions and the $\mathrm{Fe}^{5+}$ ions along the cubic [111] direction in a sequence of $\cdots \mathrm{Fe}^{3+} \mathrm{Fe}^{3+} \mathrm{Fe}^{5+} \ldots$. However, they could not observe the superlattice reflections due to the charge ordering presumably because the weak intensity of the reflections in the powder sample, although it is well known that in many charge ordered systems the ordering of charges strongly couples with lattice and produces periodic modulation in the crystal structure [1, 2, 3, $3,5,5$. Recently Li et al. observed the superlattice reflections of the charge ordering in $\mathrm{La}_{1-x} \mathrm{Sr}_{x} \mathrm{FeO}_{3}$ single crystals for the first time by electron diffraction measurements [8]. Park et al. showed that similar charge and spin ordering also exists in $R_{1 / 3} \mathrm{Sr}_{2 / 3} \mathrm{FeO}_{3}$ single crystals where $R$ is rare-earth atoms other than La [9].
Neutron diffraction is very useful to investigated the spin and charge coupled physics such as charge ordering, because it can detect the direct evidence of the magnetic ordering and the structural modulations related to the ordering of charges which is formed in a bulk sample. Therefore we performed the neutron diffraction experiments on one of the 2/3-hole-doped $\mathrm{Fe}$ oxides, $\mathrm{Nd}_{1 / 3} \mathrm{Sr}_{2 / 3} \mathrm{FeO}_{3}$. By utilizing a single crystal sample, we could find the superlattice reflections due to the structural modulations by the charge ordering together with magnetic reflections. We could also detect a subtle change of the nature of the charge ordering as a function of temperature.

A single crystal sample studied in the present study was grown by the floating zone method in oxygen atmosphere with a traveling speed of $1.0 \mathrm{~mm} / \mathrm{h}$. The detailed procedures of the sample preparation have already been described elsewhere [9]. The quality of the sample was checked by x-ray powder diffraction measurements and by electron probe microanalysis.

Neutron diffraction experiments were performed using triple axis spectrometer GPTAS installed at the JRR$3 \mathrm{M}$ reactor in JAERI, Tokai, Japan with fixed incident neutron momentums $k_{\mathrm{i}}=2.66 \AA^{-1}$ and $3.83 \AA^{-1}$. The combination of collimators were $20^{\prime}-40^{\prime}-20^{\prime}$-open and $40^{\prime}$ $80^{\prime}-80^{\prime}-80^{\prime}$ (from monochromator to detector). Although the crystal structure of the sample has a slight rhombohedral distortion along the cubic [111] direction $\left(\alpha=60.1^{\circ}\right.$ at $300 \mathrm{~K})$, we employed the cubic lattice $(a \sim 3.85 \AA)$ notation of the scattering plane. The sample was mounted in an $\mathrm{Al}$ can filled with $\mathrm{He}$ gas, and was attached to the cold head of a closed-cycle helium gas refrigerator. The temperature was controlled within an accuracy of $0.2 \mathrm{~K}$.

First we show the results of resistivity and magnetization measurements which were performed on the same crystal used in the neutron diffraction study [10]. Figure 1 shows the temperature dependence of the resistivity and the spontaneous magnetization. The resistivity at room temperature is relatively low and gradually increases as temperature decreases. However, it shows a steep increase below $T_{\mathrm{CO}}=185 \mathrm{~K}$ because of the charge ordering transition. As shown in Fig. 1, small sponta- 
neous magnetization appears below $T_{\mathrm{CO}}$, signaling the onset of the antiferromagnetic ordering with minute spin canting.

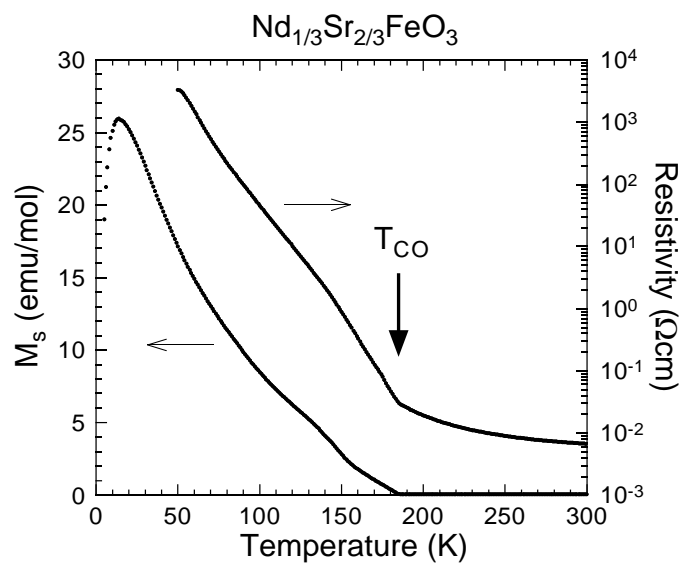

FIG. 1. Temperature dependence of resistivity and spontaneous magnetization for $\mathrm{Nd}_{1 / 3} \mathrm{Sr}_{2 / 3} \mathrm{FeO}_{3}$.

In order to characterize the charge and spin ordering, we surveyed the $(h h l)$ scattering plane and found some superlattice reflections below $T_{\mathrm{CO}}$. For an example of such survey scans, we show in Fig. 2 profiles of line scans along [11̄1] direction measured at $200 \mathrm{~K}$ and $10 \mathrm{~K}$. One can see that at $10 \mathrm{~K}$ superlattice reflections appear at $\left(\frac{1}{6}, \frac{1}{6}, \frac{5}{6}\right),\left(\frac{1}{3}, \frac{1}{3}, \frac{2}{3}\right),\left(\frac{1}{2}, \frac{1}{2}, \frac{1}{2}\right),\left(\frac{2}{3}, \frac{2}{3}, \frac{1}{3}\right)$, and $\left(\frac{5}{6}, \frac{5}{6}, \frac{1}{6}\right)$. The observed superlattice reflections can be classified into three groups according to their modulation vectors $\boldsymbol{q}: \boldsymbol{q}_{\frac{1}{6}}=a^{*}\left(\frac{1}{6}, \frac{1}{6}, \frac{1}{6}\right), \boldsymbol{q}_{\frac{1}{3}}=a^{*}\left(\frac{1}{3}, \frac{1}{3}, \frac{1}{3}\right)$, and $\boldsymbol{q}_{\frac{1}{2}}=a^{*}\left(\frac{1}{2}, \frac{1}{2}, \frac{1}{2}\right)$. Due to the twin domains of the cubic structure, we also observed reflections with $\boldsymbol{q}=a^{*}\left(\frac{1}{6}, \frac{1}{6},-\frac{1}{6}\right)$ etc. in the $(h h l)$ zone.

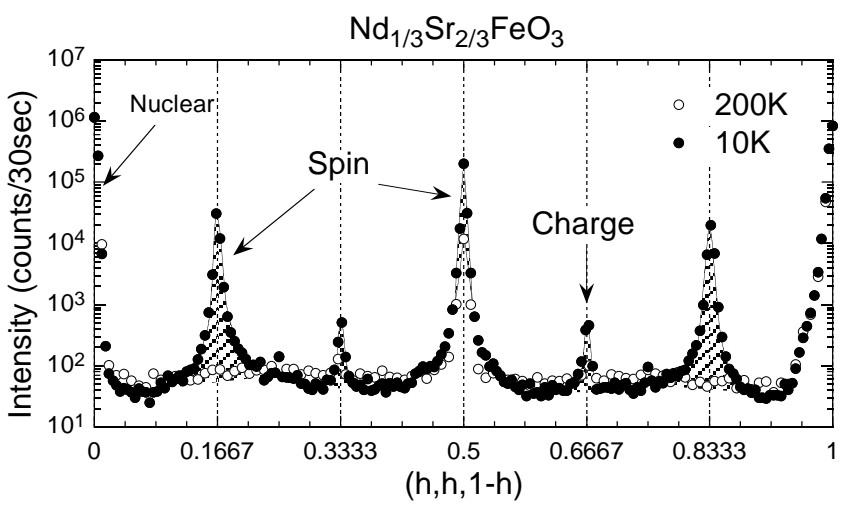

FIG. 2. A profile of the line scan along the [11̄i] direction at $200 \mathrm{~K}$ and $10 \mathrm{~K}$. Shaded peaks represent the superlattice reflections due to the magnetic and charge ordering.

$\boldsymbol{q}_{\frac{1}{3}}$ reflections were observed also by electron diffraction measurements 88.9] and they indicate lattice modulation along the cubic [111] direction whose period was three times larger than the lattice spacing of the (111) plane. This modulation should originate from the charge ordering of $\cdots \mathrm{Fe}^{3+} \mathrm{Fe}^{3+} \mathrm{Fe}^{5+} \ldots$ and accompanying local lattice distortion. The intensity of $\boldsymbol{q}_{\frac{1}{6}}$ and $\boldsymbol{q}_{\frac{1}{2}}$ reflections obey the $Q$ dependence of the magnetic form factor of the Fe ion. Therefore they can be attributed to the antiferromagnetic ordering, which is consistent with the previous powder neutron diffraction measurements on $\mathrm{La}_{1 / 3} \mathrm{Sr}_{2 / 3} \mathrm{FeO}_{3}$ [7]. Note that nuclear reflections were observed at $\left(\frac{h}{2}, \frac{h}{2}, \frac{l}{2}\right)$ even at $T>T_{\mathrm{CO}}$ (Fig. 2). These reflections are forbidden in the previously proposed rhombohedral symmetry $R \overline{3} c$, and indicate that the true crystal symmetry of the present sample is lower than $R \overline{3} c$ [7, 8,9$]$.

The existence of $\boldsymbol{q}_{\frac{1}{6}}$ and $\boldsymbol{q}_{\frac{1}{2}}$ magnetic modulation vectors means that the distribution of magnetic moments can be represented by the sum of two Fourier components, each has a wave vector of $\boldsymbol{q}_{\frac{1}{6}}$ and $\boldsymbol{q}_{\frac{1}{2}}$. As a consequence, if one assume the magnetic moments are localized on the Fe sites, there are two Fe sites in the ratio of $2: 1$ in the sequence of $\cdots \Uparrow \Downarrow \downarrow \Downarrow \Uparrow \uparrow \Uparrow \cdots$. The sites denoted by $\Uparrow$ and those by $\uparrow$ may be attributed to $\mathrm{Fe}^{3+}$ sites and $\mathrm{Fe}^{5+}$ sites, respectively.

In order to analyze the magnetic moments for two $\mathrm{Fe}$ sites, we have performed neutron powder diffraction measurements to avoid difficulty in analyzing the single crystal caused by the domain distribution. The measurement was performed at $50 \mathrm{~K}$, because below that temperature, the reflections by the ordering of the magnetic moments of $\mathrm{Nd}^{3+}$ ions superpose the $\boldsymbol{q}_{\frac{1}{6}}$ magnetic reflections (see below). The obtained value of the magnetic moments are $3.7 \mu_{\mathrm{B}}$ for $\mathrm{Fe}^{3+}$ site and $2.3 \mu_{\mathrm{B}}$ for $\mathrm{Fe}^{5+}$ site assuming the spins lie in the (111) plane. The direction of the spins in the (111) plane could not be determined due to the high symmetry. These values are similar to those obtained for $\mathrm{La}_{1 / 3} \mathrm{Sr}_{2 / 3} \mathrm{FeO}_{3}\left(3.61 \mu_{\mathrm{B}}\right.$ for $\mathrm{Fe}^{3+}$ site and $2.72 \mu_{\mathrm{B}}$ for $\mathrm{Fe}^{5+}$ site) [7]. The smaller observed magnetic moments than their nominal values of $5 \mu_{\mathrm{B}}$ and $3 \mu_{\mathrm{B}}$ indicate the strong hybridization of the Fe $3 \mathrm{~d}$ orbitals and the $\mathrm{O} 2 \mathrm{~d}$ orbitals because of the small charge-transfer energy of $\mathrm{Fe}$ oxides [11, 12].

Figure 3 shows temperature dependence of the intensity of (a) the $\boldsymbol{q}_{\frac{1}{3}}$ charge superlattice reflection, (b) $\boldsymbol{q}_{\frac{1}{6}}$ (open circles) and $\boldsymbol{q}_{\frac{1}{2}}$ (closed circles) spin superlattice reflections. As decreasing temperature, all the reflection start to develop at the same temperature $T_{\mathrm{CO}}=185 \mathrm{~K}$, indicating that charges and spins order simultaneously, which is consistent with the resistivity and magnetization data shown in Fig. 1. The transition at $T_{\mathrm{CO}}$ is first order with small hysteresis of $\sim 4 \mathrm{~K}$. The increase of the intensity of the $\left(\frac{5}{6} \frac{5}{6} \frac{5}{6}\right)$ reflection below $T \sim 50 \mathrm{~K}$ comes from the ordering of the spins of $\mathrm{Nd}^{3+}$ ions. 


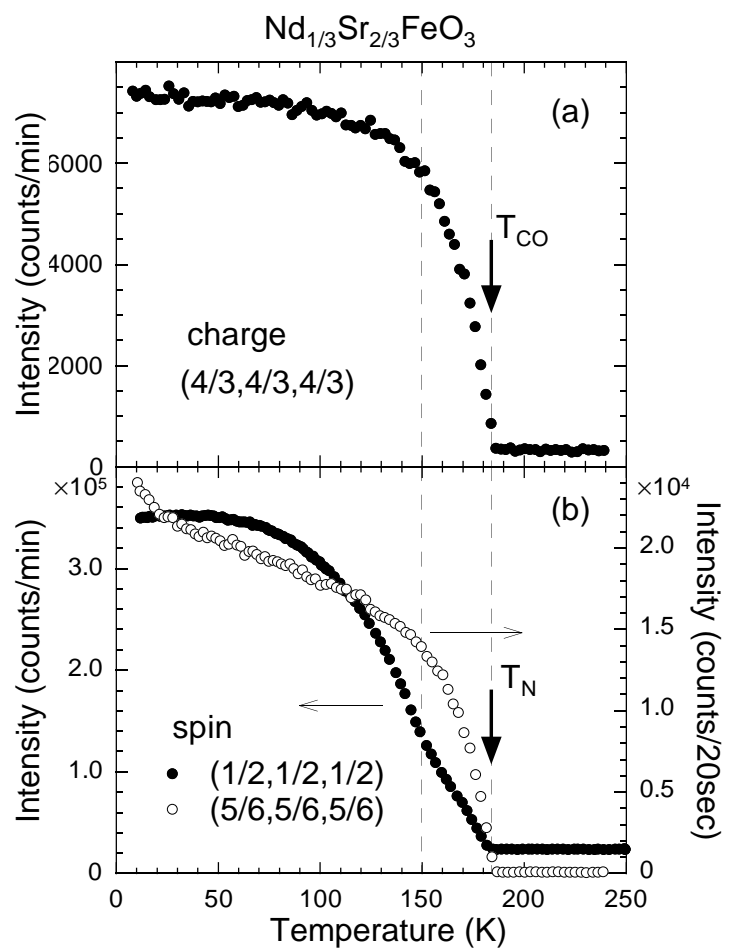

FIG. 3. Temperature dependence of the intensity of the spin and charge super lattice reflections. (a) Charge superlattice reflection $\left(\frac{4}{3} \frac{4}{3} \frac{4}{3}\right)$. (b) Spin superlattice reflection $\left(\frac{5}{6} \frac{5}{6} \frac{5}{6}\right)$ (open circles) and $\left(\frac{1}{2} \frac{1}{2} \frac{1}{2}\right)$ (closed circles).

The development of the charge ordering almost saturates around $T_{\mathrm{s}}=150 \mathrm{~K}$. The character of the spin ordering also changes at this temperature. The slope of the curve for the $\boldsymbol{q}_{\frac{1}{2}}$ reflection becomes steeper below $T_{\mathrm{S}}$, while the growth of the $\boldsymbol{q}_{\frac{1}{6}}$ reflection is slightly suppressed around $T_{\mathrm{s}}$.

The anomaly in the spin superlattice reflections at $T_{\mathrm{s}}$ can not be explained by the rotation of the spin orientations. Because the scattering vectors of the two kinds of the spin reflections shown in Fig. 3 (b) are parallel, the rotation of the spins should produce the same effect on the intensity of both spin reflections. Therefore, the anomaly at $T_{\mathrm{s}}$ should be attributed to the change of the ratio of the two Fourier component of the spin density wave at this temperature. If one assume the moments are localized at Fe sites, this means that the moments on $\mathrm{Fe}^{3+}$ sites increase while those on $\mathrm{Fe}^{5+}$ sites decrease, suggesting the enhancement of the charge disproportionation. Of course this interpretation is too naïve because the holes may locate on oxygen sites and the magnetic moments distribute continuously from site to site due to the hybridization of the Fe $3 \mathrm{~d}$ orbitals and the $\mathrm{O} 2 \mathrm{p}$ orbitals. Nevertheless, from the fact that the charge ordering almost saturates around $T_{\mathrm{s}}$, one can say that the change of the distribution of the magnetic moments are correlated with the charge ordering, and the nature of the charge and the spin ordering changes when the charge ordering sufficiently develops. We should note that we also observed the similar anomaly at $T<T_{\mathrm{CO}}$ in another 2/3-hole-doped charge disproportionated $\mathrm{Fe}$ oxides $\mathrm{Pr}_{1 / 3} \mathrm{Sr}_{2 / 3} \mathrm{FeO}_{3}$ [13].

One of the most interesting phenomena in the charge ordering transition in the hole-doped transition metal oxides is stripe order, where the doped holes align to form domain walls and spins order antiferromagnetically. Most of the stripe ordering observed so far, e.g. in $\mathrm{La}_{2-x} \mathrm{Sr}_{x} \mathrm{CuO}_{4}$ [1] or $\mathrm{La}_{2-x} \mathrm{Sr}_{x} \mathrm{NiO}_{4+\delta}$ [2, 3], is two dimensional (2 D) which has one dimensional domain walls. On the other hand, the charge and spin ordering pattern of the $2 / 3$-hole-doped cubic perovskite Fe oxides including $\mathrm{Nd}_{1 / 3} \mathrm{Sr}_{2 / 3} \mathrm{FeO}_{3}$ can be viewed as a three dimensional (3 D) stripe order propagating the [111] direction with 2 D domain walls parallel to (111) planes,

One of the clear differences between the $2 \mathrm{D}$ and the 3 $\mathrm{D}$ stripe order is the relation between the charge ordering temperature $T_{\mathrm{CO}}$ and the spin ordering temperature $T_{\mathrm{N}}$. In the $2 \mathrm{D}$ stripe order, spins always order after the charges order, while in the $3 \mathrm{D}$ stripe order, spins and charges order simultaneously. For the formation of the stripe order, the importance of the superexchange interaction between the spins as well as the Coulomb interaction between charges is widely recognized, although there is still a dispute about the driving force for the stripe order. Zachar et al. proposed a phase diagram for the stripe order based on Landau theory [15]. They claimed that there are several ways in the transition to the stripe ordered state: a charge driven transition, a charge-spin coupling driven transition, and a spin driven transition. In the charge driven transition, $T_{\mathrm{N}}$ should be lower than $T_{\mathrm{CO}}$, while in the charge-spin coupled transition and in the spin driven transition $T_{\mathrm{N}}$ should be same as $T_{\mathrm{CO}}$. Moreover, the spin-charge coupled transition should be the first order while the spin driven transition should be the second order. In their framework, the $2 \mathrm{D}$ stripe order observed in cuprates or nickelates is classified into the charge driven transition, and the $3 \mathrm{D}$ stripe order observed in the present study can be classified into the spin-charge coupling driven transition. In any event, the fact that spins and charges order simultaneously in the 3 D stripe order means that the role of the spin ordering in the stripe order becomes relatively important compared to the $2 \mathrm{D}$ stripe.

We think the increase of the relative importance of the spin ordering in the $3 \mathrm{D}$ stripe can be interpreted as a consequence of the difference in the dimensionality between the $2 \mathrm{D}$ and the $3 \mathrm{D}$ stripe. In the $2 \mathrm{D}$ stripe order, the number of the nearest holes around a hole in a domain wall becomes larger because the domain walls become 2 D. Therefore, the energy loss by the Coulomb repulsion between the holes may become larger as compared to the $3 \mathrm{D}$ stripe order. On the other hand, the number of the nearest sites for the undoped region also becomes larger because of the three dimensionality, which may increase the energy gain by the superexchange interaction between spins.

In order to verify the above scenario, the energy scale 
of the Coulomb interaction and the superexchange interaction should be examined. We would like to note that by recent Hartree-Fock calculations, it has been shown that the $3 \mathrm{D}$ stripe ordering observed in Fe oxides can be well explained by the superexchange interaction [14], which indicates the importance of the spin interaction for the formation of the stripe order.

In summary, we have investigated the charge and spin ordering in a $\mathrm{Nd}_{1 / 3} \mathrm{Sr}_{2 / 3} \mathrm{MnO}_{3}$ crystal using neutron diffraction technique. We measured the superlattice reflections due to the charge and spin ordering, and confirmed that charges and spins order simultaneously at $T_{\mathrm{CO}}=185 \mathrm{~K}$. The character of the charge and spin ordering changes at $150 \mathrm{~K}$ when the charge ordering almost saturates. The pattern of the charge and spin ordering in this sample can be viewed as the $3 \mathrm{D}$ stripe order, and the transition may be driven by the spin-charge coupling.

This work was supported by a Grant-In-Aid for Scientific Research from the Ministry of Education, Science, Sports and Culture, Japan and by the New Energy and Industrial Technology Development Organization (NEDO) of Japan.

[1] J. M. Tranquada, B. J. Sternlieb, J. D. Axe, Y. Nakamura, and S. Uchida, Nature (London) 375 (1995) 561.

[2] J. M. Tranquada, D. J. Buttrey, and V. Sachan, Phys. Rev. B 54 (1996) 12318; P. Wochner, J. M. Tranquada, D. J. Buttrey, and V. Sachan, Phys. Rev. B 57 (1998) 1066.

[3] H. Yoshizawa, T. Kakeshita, R. Kajimoto, T. Tanabe, T. Katsufuji, and Y. Tokura, Phys. Rev. B 61 (2000) R854.

[4] Y. Tomioka, A. Asamitsu, Y. Moritomo, H. Kuwahara, and Y. Tokura, Phys. Rev. Lett. 74 (1995) 5108.

[5] H. Kuwahara, Y. Tomioka, A. Asamitsu, Y. Moritomo, and Y. Tokura, Science 270 (1995) 961.

[6] M. Takano and Y. Takeda, Bull. Inst. Chem. Res., Kyoto Univ. 61 (1983) 406.

[7] P. D. Battle, T. C. Gibb, and P. Lightfoot, J. Solid State Chem. 84 (1990) 271.

[8] J. Q. Li, Y. Matsui, S. K. Park, and Y. Tokura, Phys. Rev. Lett. 79 (1997) 297.

[9] S. K. Park, T. Ishikawa, Y. Tokura, J. Q. Li, and Y. Matsui, Phys. Rev. B 60 (1999) 10788.

[10] Y. Taguchi, S. K. Park, and Y. Tokura, unpublished.

[11] A. E. Bocquet, A. Fujimori, T. Mizokawa, T. Saitoh, H. Namatame, S. Suga, N. Kimizuka, Y. Takeda, and M. Takano, Phys. Rev. B 45 (1992) 1561.

[12] J. Matsuno, T. Mizokawa, and A. Fujimori, K. Mamiya, Y. Takeda, S. Kawasaki, and M. Takano, Phys. Rev. B 60 (1999) 4605.

[13] Y. Oohara, M. Kubota, H. Yoshizawa, S. K. Park, and Y. Tokura, unpublished.

[14] T. Mizokawa and A. Fujimori, Phys. Rev. Lett. 80 (1998) 1320.

[15] O. Zachar, S. A. Kivelson, and V. J. Emery, Phys. Rev. B 